\title{
Features of public open spaces and physical activity among children: findings from the CLAN Study
}

\author{
Anna Timperio $^{1}$, Billie Giles-Corti ${ }^{2}$, David Crawford ${ }^{1}$, \\ Nick Andrianopoulos ${ }^{1}$, Kylie Ball ${ }^{1}$, Jo Salmon ${ }^{1}$, Clare Hume ${ }^{1}$ \\ ${ }^{1}$ Centre for Physical Activity and Nutrition Research, Deakin University \\ ${ }^{2}$ School of Population Health, University of Western Australia
}

Corresponding author:

Dr Clare Hume

Centre for Physical Activity and Nutrition Research

Deakin University

221 Burwood Highway

Burwood VIC 3125, Australia

Telephone: +61 (0)39251 7723

Facsimile: +61 (0)3 92446017

Email: clare.hume@deakin.edu.au 


\begin{abstract}
Objective: To examine associations between features of public open spaces, and children's physical activity.
\end{abstract}

Participants: 163 children aged 8-9 years and 334 adolescents aged 13-15 years from Melbourne, Australia participated in 2004.

Methods: A Geographic Information System was used to identify all public open spaces (POS) within $800 \mathrm{~m}$ of participants' homes and their closest POS. The features of all POS identified were audited in 2004/5. Accelerometers measured moderate-tovigorous physical activity (MVPA) after school and on weekends. Linear regression analyses examined associations between features of the closest POS and participants' MVPA.

Results: Most participants had a POS within $800 \mathrm{~m}$ of their home. The presence of playgrounds was positively associated with younger boys' weekend MVPA (B=24.9 mins/day; $\mathrm{p} \leq 0.05$ ), and lighting along paths was inversely associated with weekend MVPA ( $B=-54.9$ mins/day; $p \leq 0.05)$. The number of recreational facilities was inversely associated with younger girls' MVPA after school (B=-2.6 mins/day; $p \leq 0.05)$ and on the weekend $(B=-8.7$ mins/day; $p \leq 0.05)$. The presence of trees providing shade (5.8 mins/day, $\mathrm{p} \leq 0.01)$ and signage regarding dogs $(\mathrm{B}=6.8 \mathrm{mins} /$ day, $\mathrm{p} \leq 0.05$ ) were positively associated with adolescent girls' MVPA after school.

Conclusion: Certain features of POS were associated with participants' MVPA, although mixed associations were evident. Further research is required to clarify these complex relationships.

Key words: physical activity, environment, youth 


\section{Introduction}

It is generally recognised that children should engage in at least one hour of moderate-to-vigorous physical activity (MVPA) every day (Salmon and Timperio 2007). Although little data exists to determine if children are meeting these guidelines (Salmon and Timperio 2007), in light of rapidly increasing rates of childhood obesity across the world (Wang and Lobstein 2006), the promotion of physical activity among children is an important public health priority. Social-ecological models of health behaviour suggest that the environment in which children are nested may be important influences on their physical activity (Davison and Birch 2001; Salmon and King 2005). There is support for this notion in the literature, given that several aspects of the built and social environments have been associated with physical activity, including access or availability of facilities for physical activity such as formal parks and public open spaces (Davison and Lawson 2006).

Research has shown that many adults who visit parks engage in active recreation (mobile activities or sports) whilst there (Godbey et al. 2005). Parks and public open spaces may also be important venues for children to engage in active play and other types of more formal physical activity (Bedimo-Rung et al. 2005) and are important destinations to which children walk or cycle. In an experimental study, Epstein et al. (Epstein et al. 2006) showed that when children's access to sedentary pastimes was decreased, greater access to parks was associated with a greater increase in physical activity. Studies have also shown that total area of parks relative to residential areas are positively associated with 4-7 year-old children's physical activity (Roemmich et al. 2006), a lack of nearby parks is negatively associated with 10-12 year-old girls' walking and cycling (Timperio et al. 2004), and density of parks within one mile is 
associated with adolescent girls' non-school physical activity (Cohen et al. 2006). Similar findings have been documented among adults (Giles-Corti and Donovan 2003; Troped et al. 2003; Wendel-Vos et al. 2004; Duncan and Mummery 2005). Although the presence and number of parks nearby appears to be an important correlate of physical activity among youth, little is known about the specific park characteristics that are related to physical activity (Bedimo-Rung et al. 2005; BedimoRung et al. 2006; Saelens et al. 2006).

Qualitative work with parents of elementary school-aged children suggests that features of nearby parks that are capable of satisfying children's needs might be an important correlate of children's park use (Veitch et al. 2006). Cohen et al. (Cohen et al. 2006) examined a range of features of parks near adolescent girls' homes and found that girls with parks with playgrounds, drinking fountains, streetlights, basketball courts, floodlights, multi-purpose room, a walking path, lawn games, a running track and a skateboard area within a half mile, accumulated more MVPA ( $\geq 3$ METS) than other girls. The presence of basketball courts, floodlights and a swimming area were positively associated with more intense physical activity among these girls.

In summary, few studies have sought to investigate relationships between specific features of parks and open spaces and physical activity among youth. Features of public open spaces that may be important for physical activity are likely to differ among children and adolescents. The aim of this study was to examine associations between features of public open spaces and physical activity among children aged 8-9 and $13-15$ years. 


\section{Methods}

This study drew on cross-sectional data collected in the first follow-up (in 2004) of the Children Living in Active Neighbourhoods (CLAN) study, which included 188 families of 5-6 and 402 families of 10-12 year-old children who had participated in a study of family influences on physical activity in 2001. The baseline sample was recruited from 19 state elementary schools in high and low socioeconomic areas of metropolitan Melbourne (Timperio et al. 2004; Telford et al. 2005; Timperio et al. 2005). This paper is based on the participants for whom residential address was known and usable accelerometer data were available at follow-up ( $87 \%$ of younger and $83 \%$ of older participants).

\section{Procedure}

Parents completed a questionnaire at home and each participant's physical activity was objectively-assessed using accelerometry. Residential addresses were geocoded in a Geographic Information System (GIS) and for each address the closest free or reserved access public open space (POS) along the road network, excluding educational institutions and golf courses, was identified from the Open Space 2002 spatial dataset (provided by the Australian Research Centre for Urban Ecology). Trained auditors $(\mathrm{n}=18)$ visited each POS identified to audit their features.

\section{$\underline{\text { Measures }}$}

Moderate-to-vigorous intensity physical activity

Each child was asked to wear a Manufacturing and Technologies, Inc (MTI) accelerometer on their right hip for one week (Actigraph Model AM7164-2.2C). The accelerometers were programmed to collect data on accelerations of the body in one 
minute epochs. Data were downloaded and entered into a software program to extract minutes of MVPA, consistent with children's physical activity guidelines (Salmon and Timperio 2007), at different times of the day. Cutpoints used to identify MVPA (defined as $\geq 3$ metabolic equivalents of rest, METS) were based on an established regression equation (Trost et al. 1998). Total duration (minutes) of MVPA was calculated for each weekday during after-school hours (from the last school bell until 9pm) and weekend day (6am to 9pm). Average minutes/day of MVPA after school (based on $\geq 4$ weekdays) and average minutes/day of MVPA on weekend days (based on $\geq 1$ day) were computed excluding the first and the final day of data collection and any days on which total movement counts were $<10,000$ and $\geq 20$ million and vigorous-intensity activity exceeded six hours (Telford et al. 2005). Overall, 98\% of children who agreed to wear an accelerometer at follow-up met these criteria on $\geq 4$ days and $89 \%$ on 6 days.

\section{Public open space audit}

A short audit tool was developed to assess specific features of the POS hypothesized to be important influences on children's physical activity (Crawford et al. 2008). Auditors recorded the following features: the number of recreational facilities (e.g. number of courts and ovals, athletics facilities, swimming pools and informal recreations spaces such as grassed areas); number of playgrounds; number of amenities present (e.g. rubbish bins, barbecue facilities, toilets, and drinking fountains/taps); presence of walking paths; presence of cycling paths; presence of lighting along the paths; presence of trees providing shade; presence of a water feature; presence of signs about dogs in the space (e.g. no dogs allowed, dogs only allowed on leashes, dogs allowed off leashes); and presence of signage restricting 
other activities (e.g. no ball games, no walking on the grass). The tool's inter-rater reliability, tested on 19 randomly selected POS each assessed by 10 auditors, was good $(\mathrm{k}=0.65)$. Ten auditors reassessed 19 of these POS at least one week later and intra-rater reliability was excellent (ICC or $\%$ agreement $>80 \%$ for all items).

\section{Data analysis}

The data were analysed using Stata 8 (Stata Corp, College Station TX, 2003).

Descriptive statistics were used to examine the demographic characteristics of the sample, and the characteristics of their closest POS. Independent t-tests examined differences in after-school and weekend MVPA by sex and age. Separate linear regression analyses examined associations between each feature of the closest POS and after-school and weekend MVPA, adjusting for clustering by school (baseline unit of recruitment) using Stata's cluster command (e.g., regress y x1, cluster(school).

\section{Results}

\section{$\underline{\text { Sample characteristics }}$}

The demographic characteristics of the sample are shown in Table 1. Participants were 163 children aged approximately 9 years, and 334 adolescents aged approximately 14 years.

\section{Moderate-to-vigorous intensity physical activity}

Table 1 also shows the average duration of MVPA accumulated after school and on the weekend. On average, younger children spent approximately twice as much time after school $(\mathrm{p} \leq 0.0001)$ and $2.5-3$ times as much time on weekends $(\mathrm{p} \leq 0.0001)$ performing MVPA compared to adolescent boys and girls. Among adolescents, boys 
performed more MVPA than girls after school (approximately 9 minutes/day difference, $\mathrm{p}=0.0002$ ) and on weekends (approximately 22 minutes/day difference, $\mathrm{p} \leq 0.0001)$. There were no significant sex differences among younger children.

Insert Table 1 about here

\section{$\underline{\text { Public open space features }}$}

Participants lived approximately $300 \mathrm{~m}$ (mean=299.8m; SD=245.6m) from their closest POS. Table 2 shows the distribution of features of the closest POS. On average, there was at least one recreational facility (e.g. court, oval, informal grassed area) and more than two amenities (e.g. toilets, drinking fountains/taps) within each POS. Walking paths were present in approximately two-thirds and cycling paths in half of the POS. Trees providing shade were also present in most POS (61\%), but few had lighting along paths (15\%) or water features (17\%). Signage regarding dogs was present in about $30 \%$ of the POS, while signage restricting other activities was present in $20 \%$.

Insert Table 2 about here

$\underline{\text { POS features and physical activity }}$

Table 3 shows associations between POS features and children's MVPA. There were no associations between any features of the child's closest POS and younger boys' 
MVPA after school. Among younger girls, the number of recreational facilities present was inversely associated with MVPA after school. On weekends, younger boys performed $25 \mathrm{mins}$ /day more MVPA with each additional playground in their closest POS. In contrast, they did less MVPA on the weekend compared to other boys if their closest POS had lighting along the paths. Among younger girls, the number of recreational facilities was inversely associated with weekend MVPA.

There were no associations between any features of the closest POS and adolescent boys' MVPA after school. However, adolescent girls did more MVPA after school if their closest POS had trees that provided shade and had signage regarding dogs, compared with those whose closest POS did not have these features. There were no significant associations between the features of the closest POS and adolescents boys' or girls' MVPA on the weekend.

Insert Table 3 about here

\section{Discussion}

This study examined associations between POS features and physical activity among Australian children aged 8-9 and 13-15 years. This is one of the first studies to examine such associations among children, and the objective assessment of physical activity and the use of a reliable audit tool are strengths. Although some POS features were associated with MVPA after school and on weekends, the findings were inconsistent across age and sex groups. This may reflect differences in availability of 
free time, lifestyle and interests between children and adolescents. However, it is encouraging that, on average, the children in this study lived relatively close to a POS (approximately 300m); well within what parents consider a walking distance for their child (Timperio et al. 2004). This is consistent with other Australian research where over $90 \%$ of children reported that they could walk or cycle to their local park (Hume et al. 2007) and may reflect planning guidelines about the positioning of parks and other open spaces in some parts of Australia (Leary and McDonnell 2001).

Playgrounds were positively associated with younger boys' physical activity, but there was only a non-significant trend $(\mathrm{p}=0.067)$ among adolescent boys despite the larger sample. This may reflect the lessened importance of playgrounds among boys as they become older. There were no significant associations for playgrounds among girls. This contrasts previous research among U.S. adolescent girls which included all parks within one mile of girl's homes (Cohen et al. 2006). One explanation for these contrasting findings may be that the closest POS (as included in the present study) may not be the space that is most interesting or appealing for girls or is visited regularly; other POS may be visited instead. Further, if many boys are visiting and playing in playgrounds, girls may not feel inclined to enter or use the playground in their closest park.

POS features that were positively associated with adolescent girls' physical activity after school in the present study included the presence of trees providing shade and signage regarding dogs. This suggests that these sorts of features may be more appealing for older girls who are perhaps engaging in non-organised activities, rather than organised or planned physical activity. These findings highlight the importance 
of the availability of a range of features in POS that appeal to boys and girls across the age spectrum. The inclusion of age-appropriate and appealing playgrounds may be particularly important among boys.

Contrary to expectations, several features of the closest POS were inversely associated with children's MVPA. Although the presence of lighting could be considered a safety feature, this item was negatively associated with weekend physical activity among younger boys. This may reflect the types of POS that require lighting (e.g., trouble spots or POS with formal recreation options, such as sporting pitches, ovals and bicycle paths, used by the public at night) that young boys may not be allowed or interested to visit. In addition, the number of formal recreation options was inversely associated with physical activity among young girls. This may be because girls in this age group might prefer less formal play options. Further, the types of recreation options offered may be more suitable to older children or adults, catering for structured sports rather than other types of play or recreation, though the lack of association with MVPA among the other groups studied suggests that characteristics of facilities may be more important than quantity.

Overall, few features of children's closest POS were associated with their MVPA on weekdays or weekends. In light of the mixed and unexpected findings, it could be hypothesised that the features of the child's 'closest' POS may be less relevant than the quality of these features, which was not measured in this study. Previous research suggests that children are venturing further into their local neighbourhood to access more desirable parks, with the park usually visited located on average up to twice the distance from home than the closest park (Veitch et al. in press). In only considering 
the 'closest' POS, the present study may not have included POS visited by participants. Further, this study did not consider whether the closest POS was easily accessible. The need to cross roads and a lack of infrastructure to aid safe road crossings, for example, are important barriers to children's walking and cycling (Timperio et al. 2004) and may inhibit visits to POS. In addition, we did not measure physical activity specifically undertaken within POS; it is possible that none or little of the MVPA measured took place within POS. Future research could examine which features are associated within physical activity specifically undertaken during visits to POS. This study was also limited by a small sample size, particularly among the younger cohort.

In summary, our findings suggest that some features of the closest POS, particularly playgrounds, are associated with children's physical activity. To better understand these relationships, future studies should consider objectively assessing the features of POS actually frequented by children, as well as the quality and accessibility of these and other POS close to home. Future urban planning and design of POS should consider incorporating interesting and age-appropriate playground equipment and POS features to promote children's physical activity.

\section{Acknowledgements}

This study was funded by a grant from the National Health and Medical Research Council (NHMRC, ID: 274309). Thanks to Emma Shorter, Dino Zanon and Leah Galvan for their contributions to the development of the audit instrument. Thanks also to Michelle Jackson, Sophie Thal-Jantzen and Leah Galvan for coordinating data collection. Anna Timperio, and David Crawford are supported by Public Health 
Research Fellowships from the Victorian Health Promotion Foundation. Jo Salmon is supported by a Heart Foundation and sanofi-aventis Career Development Award Billie Giles-Corti and Kylie Ball are supported by Career Development Awards from the National Heart Foundation and the NHRMC.

\section{References}

Bedimo-Rung, A. L., J. Gustat, et al. (2006). "Development of a direct observation instrument to measure environmental characteristics of parks for physical activity." Journal of Physical Activity and Health 3: S176-S189.

Bedimo-Rung, A. L., A. J. Mowen, et al. (2005). "The significance of parks to physical activity and public health: a conceptual model." American Journal of Preventive Medicine 28(2 Suppl 2): 159-68.

Cohen, D. A., J. S. Ashwood, et al. (2006). "Public parks and physical activity among adolescent girls." Pediatrics 118(5): e1381-9.

Crawford, D., A. Timperio, et al. (2008). "Do features of public open spaces vary according to neighbourhood socio-economic status?" Health \& Place 14: 887891.

Davison, K. K. and L. L. Birch (2001). "Childhood overweight: a contextual model and recommendations for future research." Obesity Reviews 2(3): 159-71.

Davison, K. K. and C. T. Lawson (2006). "Do attributes in the physical environment influence children's physical activity? A review of the literature." International Journal of Behavioral Nutrition and Physical Activity 3: 19.

Duncan, M. and K. Mummery (2005). "Psychosocial and environmental factors associated with physical activity among city dwellers in regional Queensland." Preventive Medicine 40(4): 363-72.

Epstein, L. H., S. Raja, et al. (2006). "Reducing sedentary behavior: the relationship between park area and the physical activity of youth." Psychological Science 17(8): 654-9.

Giles-Corti, B. and R. J. Donovan (2003). "Relative influences of individual, social environmental, and physical environmental correlates of walking." American Journal of Public Health 93(9): 1583-9.

Godbey, G. C., L. L. Caldwell, et al. (2005). "Contributions of leisure studies and recreation and park management research to the active living agenda." American Journal of Preventive Medicine 28(2 Suppl 2): 150-8.

Hume, C., J. Salmon, et al. (2007). "Associations of children's perceived neighborhood environments with walking and physical activity." American Journal of Health Promotion 21(3): 201-7.

Leary, E. and M. McDonnell (2001). "Quantifying Public Open Space in metropolitan Melbourne." Parks and Leisure 4: 34-36. 
Roemmich, J. N., L. H. Epstein, et al. (2006). "Association of access to parks and recreational facilities with the physical activity of young children." Preventive Medicine 43(6): 437-41.

Saelens, B. E., L. D. Frank, et al. (2006). "Measuring physical environments of parks and playgrounds: EAPRS Instrument development and reliability." Journal of Physical Activity and Health 3(S190-S207).

Salmon, J. and A. C. King (2005). Population approaches to increasing physical activity among children and adults. Obesity prevention in the 21 st century: Public health approaches to tackle the obesity pandemic. D. Crawford and R. Jeffrey. Oxford, Oxford University Press: 129-152.

Salmon, J. and A. Timperio (2007). "Prevalence, trends and environmental influences on child and youth physical activity." Medicine and Sports Science 50: 18399.

Telford, A., J. Salmon, et al. (2005). "Examining physical activity among 5- to 6- and 10- to 12-year-old children: The Children's Leisure Activities Study." Pediatric Exercise Science 17: 266-280.

Timperio, A., D. Crawford, et al. (2004). "Perceptions about the local neighborhood and walking and cycling among children." Preventive Medicine 38(1): 39-47.

Timperio, A., J. Salmon, et al. (2005). "Perceptions of local neighbourhood environments and their relationship to childhood overweight and obesity." International Journal of Obesity 29(2): 170-5.

Troped, P. J., R. P. Saunders, et al. (2003). "Correlates of recreational and transportation physical activity among adults in a New England community." Preventive Medicine 37(4): 304-10.

Trost, S. G., D. S. Ward, et al. (1998). "Validity of the computer science and applications (CSA) activity monitor in children." Medicine and Science in Sports and Exercise 30(4): 629-33.

Veitch, J., S. Bagley, et al. (2006). "Where do children usually play? A qualitative study of parents' perceptions of influences on children's active free-play." Health \& Place 12(4): 383-93.

Veitch, J., J. Salmon, et al. (in press). "Children's active free-play in local neighborhoods: A behavioral mapping study." Health Education Research.

Wang, Y. and T. Lobstein (2006). "Worldwide trends in childhood overweight and obesity." International Journal of Pediatric Obesity 1(1): 11-25.

Wendel-Vos, G. C., A. J. Schuit, et al. (2004). "Factors of the physical environment associated with walking and bicycling." Medicine and Science in Sport and Exercise 36(4): 725-30. 


\section{Precis}

Several features of public open spaces, were associated with children's $(n=497)$ physical activity, specifically playgrounds among boys'. Urban planners should ensure public open spaces contain age and sex appropriate features. 